\title{
Glottopol
}

Revue de sociolinguistique en ligne

$35 \mid 2021$

La langue à l'école, de l'institution à la classe : quelles conceptions, quelles normes, pour quels usages?

\section{Des conceptions scolaires de la langue en tension : l'exemple du programme de 2015 pour l'école primaire et le collège}

Catherine Delarue-Breton

\section{OpenEdition}

Journals

Édition électronique

URL : https://journals.openedition.org/glottopol/283

DOI : $10.4000 /$ glottopol.283

ISSN : 1769-7425

Éditeur

Presses universitaires de Rouen et du Havre

Référence électronique

Catherine Delarue-Breton, « Des conceptions scolaires de la langue en tension : l'exemple du programme de 2015 pour l'école primaire et le collège », Glottopol [En ligne], 35 | 2021, mis en ligne le 01 janvier 2021, consulté le 02 octobre 2021. URL : http://journals.openedition.org/glottopol/283 DOI : https://doi.org/10.4000/glottopol.283 


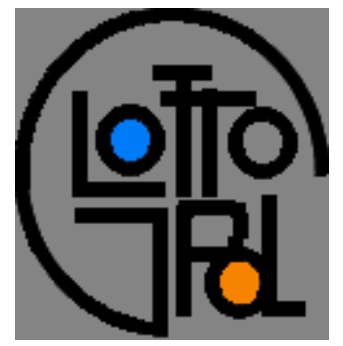

\section{GLOTTOPOL}

Revue de sociolinguistique en ligne $\mathrm{n}^{\circ} 35$ - janvier 2021

\section{La langue à l'école, de l'institution à la classe : quelles conceptions, quelles normes, pour quels usages?}

\section{SOMMAIRE}

Catherine Delarue-Breton et Élisabeth Bautier : Introduction

Catherine Delarue-Breton : Des conceptions scolaires de la langue en tension : l'exemple du programme de 2015 pour l'école primaire et le collège

Véronique Miguel-Addisu : "Sport de riche je l'ai changé en sport élitiste » : plurilinguisme et variation dans la langue de l'école du point de vue des élèves

Claire Colombel-Teuira, Véronique Fillol : Variation et approche polynomique : pour une conception plurielle de la langue à l'école calédonienne

Daphné Bloch : Pratiques langagières, situations pédagogiques et construction d'inégalités d'apprentissage à Madagascar

Samatar Abdallah Doualeh : Le français langue de l'école djiboutienne ou l'hégémonie de la norme exogène

Élise Vinel, Élisabeth Bautier : Des discours des élèves sur l'orthographe aux pratiques des enseignants, analyse d'entretiens métagraphiques

Thierry Pagnier, Belinda Lavieu-Gwozdz: Regards sur le discours scolaire: saisir des conceptions de la langue et de son enseignement

Jacques Crinon, Georges Ferone, Hélène Font : Les enseignants et l'orthographe, une enquête au cycle 3

Christel Troncy : Les enseignants, la norme scolaire et la pluralité langagière dans deux écoles immersives à programme français en Californie. Dynamiques des attitudes et des pratiques lors d'une recherche collaborative

Erwan Le Pipec: L'école, planche de salut du breton?

Argia Olçomendy : La langue basque dans les instructions officielles (1976-2019)

\section{Compte rendu de lecture}

Par Katrin Pfadenhauer: Ursula Reutner (Ed.), 2017, Manuel des francophonies, Berlin/Boston, de Gruyter, 745 p.

Par Robert Fournier: Shana Poplack, 2018, Borrowing: Loanwords in the speech community and in the grammar. New York: Oxford University Press ; xxi, $246 \mathrm{p}$.

Par Marie-Jeanne Verny: Catherine Adam, 2020, Bilinguisme scolaire. Familles, écoles, identités en Bretagne, éd. Peter Lang, coll. «Langue, multilinguisme et changement social », Berlin. 


\title{
DES CONCEPTIONS SCOLAIRES DE LA LANGUE EN TENSION : L'EXEMPLE DU PROGRAMME DE 2015 POUR L'ÉCOLE PRIMAIRE ET LE COLLÈGE
}

\author{
Catherine Delarue-Breton
}

Université de Rouen-Normandie

\section{Introduction}

Cette contribution propose d'abord d'identifier, dans une approche qualitative, des conceptions de la langue et de ses usages, susceptibles de traverser implicitement, et sans doute pour partie à l'insu de ses rédacteurs, le programme français de 2015, appliqué à partir de la rentrée scolaire 2016 à l'école primaire et au collège (MEN, 2015). Dans un second temps, on mettra en évidence la prégnance, dans ce même programme, d'un usage spécifique de la langue, qui prend pour thème l'explicitation. Par conceptions, nous entendons un ensemble informel de normes et de valeurs susceptibles de faire système, portant simultanément sur les fonctions et fonctionnements de la langue, mais aussi sur ses usages, et en particulier à l'école, sur les rapports que la langue peut entretenir avec les apprentissages.

Il s'agit ainsi d'étudier ce document d'une part en procédant à une analyse discursive de l'usage des termes langue et langage, ainsi que de leurs dérivés, en examinant leur contexte d'emploi ; d'autre part d'étudier systématiquement les 61 occurrences du terme explicite et ses dérivés (expliciter, explicitation, explicitement), et leur contexte d'emploi.

Notre étude s'inscrit dans la suite des travaux qui prennent pour objet les facteurs qui contribuent au développement, au sein même de l'école, des inégalités d'apprentissage (Bautier \& Rayou, 2009; Rochex \& Crinon, 2011 ; Delarue-Breton, 2012), et qui mettent en avant le caractère opaque, pour une partie importante des élèves, des réquisits et des situations scolaires.

L'emploi récurrent du terme explicite et de ses dérivés dans ce programme suggère en effet une volonté de prise en charge de cette opacité, et une volonté de promouvoir une plus grande transparence de ces réquisits de la part de l'institution. Pour autant, les conditions d'une réelle appropriation des enjeux des activités scolaires par tous les élèves sont multifactorielles, et supposent une clarification, déjà, de ce que recouvre la notion d'explicitation. Cela suppose également de clarifier ce qui peut et doit être explicite ou explicité, et ce qui gagnerait à ne pas l'être, du moins pas a priori, ménageant ainsi la possibilité pour les élèves de se frayer un chemin proprio motu (Brousseau, 1998) dans l'appropriation de savoirs scolaires au sens large. Or, au sein même de ce programme, on observe une pluralité et une hétérogénéité des emplois, susceptible de contribuer paradoxalement à brouiller, pour les enseignants, la perception des enjeux et visées de l'explicitation en classe. 


\section{Le programme de 2015 : maitrise de la langue et/ou langage réflexif ?}

La question de la langue et de son enseignement est simultanément une question sensible et récurrente, comme l'atteste le nombre considérable de contributions scientifiques qui se sont penchées sur la question depuis plus de vingt ans, et pour ne donner qu'un exemple, les cinq numéros de la revue Le Français Aujourd'hui qui lui ont été consacrés en dix ans, entre 2007 et $2017: \mathrm{n}^{\circ} 156$ (Enseignement de la langue : crise, tension ?), $\mathrm{n}^{\circ} 162$ (Descriptions de la langue et enseignement), $\mathrm{n}^{\circ} 173$ (Continuités et ruptures dans l'enseignement de la langue), ${ }^{\circ} 192$ (Enseigner la grammaire: contenu linguistiques et enjeux didactiques) et $\mathrm{n}^{\circ} 198$ (Nouveaux programmes et étude de la langue).

\section{La langue dans les programmes : quelques éléments d'histoire}

Du côté de l'institution, la question de la langue est tout aussi sensible et récurrente, et les programmes se renouvèlent ou visent à se renouveler à un rythme soutenu. Pour ce qui est de l'école primaire, notons simplement que les programmes successifs du début du XXI siècle, à savoir le programme de 2002, celui de 2008, puis celui de 2015, qui a fait l'objet d'« ajustements » en 2018, font apparaitre un double mouvement évolutif, qui prend d'une part appui sur les apports successifs de la recherche, dans une progression que l'on pourrait qualifier de linéaire, mais qui s'inscrit également dans un mouvement de balancier, privilégiant tantôt une approche traditionnelle, tantôt une approche plus contemporaine de la langue, de son enseignement et de ses usages. En 2002 par exemple, la priorité est donnée à l'observation réfléchie de la langue, dans une approche renouvelée,

qui préconise une construction des savoirs par un élève actif, qui manipule et classe les unités linguistiques pour dégager des unités et établir des règles. [...] Le terme de régularité est très fréquent dans tous les domaines d'étude de la langue, alors que les "phénomènes irréguliers ou exceptionnels ne relèvent pas d'un enseignement, mais, s'ils sont fréquents dans l'usage, d'un effort de mémorisation" (Pellat, $2017: 21$ ).

En 2008, il s'agit de revenir à une approche plus traditionnelle, centrée sur l'appropriation par les élèves d'une terminologie grammaticale classique, et qui se caractérise

entre autre, par un empilement quantitatif de notions à acquérir du Cours préparatoire ( $C P$ - lère primaire) à la Troisième et par l'absence de démarches d'enseignement préconisées [...]. (op. cit. : 15).

En 2015, les contenus enseignés font l'objet d'une plus grande articulation, entre eux et entre les cycles, dans une approche qui «renoue avec l'observation réfléchie de la langue des programmes du primaire de $2002 »(i b i d$.$) , et qui vise « une limitation de la terminologie$ grammaticale » (op. cit. : 17).

Enfin, en 2018, les «ajustements » apportés au programme de 2015 visent d'abord un recentrage sur ce qui est désigné comme les «fondamentaux », impliquant notamment en français un retour de la terminologie grammaticale traditionnelle, qui n'apparaissait pas dans le programme de 2015 : les termes nature et fonction des mots, COD ${ }^{1}, C O I, C C$, attribut du sujet, épithète par exemple.

Le programme qui fait l'objet de notre attention ici est celui de 2015 ; toutefois, sans proposer une comparaison systématique entre les deux programmes, nous prendrons le programme de 2008 comme point d'appui de notre étude de celui de 2015, et en ferons valoir

\footnotetext{
${ }^{1}$ COD : complément d'objet direct ; COI : complément d'objet indirect ; CC : complément circonstanciel.
} 
les points de continuité et de rupture. Cela nous permettra de donner à voir une évolution des conceptions de la langue allant au-delà des éléments abordés précédemment.

Nous précisons ici, au plan méthodologique, que nous avons retenu dans le B.O. de 2008, qui présente le programme d'enseignement pour l'école primaire (maternelle et élémentaire), l'ensemble du document, soit 39 pages. Afin de travailler sur des données à peu près comparables, sinon en volume ${ }^{2}$, du moins en termes de public visé, nous avons retenu pour le programme de 2015 d'une part le programme spécifique concernant l'école maternelle, soit 19 pages, ainsi que les pages 1 à 214 pour le programme d'enseignement pour l'école et le collège, qui intègre en outre la première classe de l'enseignement secondaire, la classe de $6^{\text {ème }}$ (les pages suivantes, c'est-à-dire 215 à 388 , qui concernent le cycle 4 , constitué des classes de $5^{\text {ème }}, 4^{\text {ème }}$ et $3^{\text {ème }}$, ont été supprimées ${ }^{3}$ ).

Le programme de 2008 était clairement focalisé sur les dimensions normatives de la langue, qu'il s'agisse des normes du système linguistique (je dis vs *je disons), appelées aussi normes structurelles, ou de normes d'usage standard (je ne veux pas vs je veux pas), sans que la distinction entre celles-ci soit par ailleurs explicitée. On observe ainsi 27 occurrences du terme correct ou de ses dérivés (correctement, correction) pour désigner la langue et ses usages, comme dans "s'exprimer de façon correcte » ou «formuler correctement des questions » (p.31), «phrases correctes» (p.29), «phrases [...] correctement construites » (p.29) ou « correction syntaxique » (p.22), « correction rédactionnelle » (p.21).

Ces formulations renvoient à ce que Dominique Bucheton (2014 : 11-12) désigne comme " paradigme de la maitrise de la langue », où le terme de paradigme ne désigne pas, dans cette acception, un modèle scientifique, mais renvoie plutôt à une conception prégnante et formelle de la langue et de ses usages à l'école, qu'elle qualifie, en l'occurrence, de "paradigme archaïque, inapproprié » (ibid.). Ce point de vue, qui ne remet pas en question, selon nous, l'intérêt porté à la langue, dénonce la centration de l'institution et des pratiques pédagogiques scolaires sur ses normes, qu'il s'agisse des normes du système linguistique ou des normes d'usage standard, au détriment de ses usages conceptuels, et donc au détriment des discours des disciplines qui permettent aux élèves de s'approprier les notions, démarches ou raisonnements attendus, autrement dit de s'approprier des catégories de pensée visant à instrumenter leur relation au monde.

$\mathrm{Ce}$ «paradigme de la maitrise de la langue » focalise ainsi l'attention des enseignants et des élèves sur une norme qui s'inscrit dans une conception normalisante, où la norme relève d'une imposition sociale, plutôt que dans une conception normative, pour reprendre la distinction de Laurence Cornu (2009), où la norme a pour visée de favoriser la compréhension des usages langagiers nécessaires pour s'approprier notions et concepts disciplinaires, et aller au-delà des procédures routinisées des tâches. Ainsi, la norme linguistique, notamment à l'école, se voit implicitement tendue entre "prétention au bon usage» et "outil cognitif puissant» (Ramognino, 2007). Si notre point de vue n'est pas d'opposer maitrise de la langue et appropriation du discours scolaire, il s'agit cependant d'une part d'opter résolument pour un rééquilibrage en faveur du discours, autrement dit en faveur de l'outil cognitif puissant, d'autre part de proposer une mise en évidence de cette tension, dont on peut penser qu'elle serait sans doute moins clivante et peut-être même structurante si les enseignants pouvaient s'en emparer.

Dans le programme de 2015, le terme correct, ou ses dérivés, apparait seulement dans 4 occurrences pour désigner la langue et ses usages (contre 27 occurrences, dans le programme de 2008, pourtant dix fois moins volumineux).

\footnotetext{
${ }^{2}$ Ce programme de 2015, qui comprend 388 pages, est en effet beaucoup plus volumineux que celui de 2008 , qui n'en comprend que 39 .

${ }^{3}$ L'école primaire française, qui accueille les élèves de 3 à 11 ans, est composée de l'école maternelle et de l'école élémentaire. L'école élémentaire commence au Cours Préparatoire (ou CP) ; le collège (classes de $6^{\text {ème }}, 5^{\text {ème }}, 4^{\text {ème }}$ et $3^{\text {ème}}$ ) accueille les élèves de 11 à 15 ans. L'école primaire et le collège recouvrent la scolarité obligatoire.
} 


\section{Le B.O. de 2015 : une conception revisitée?}

Il faut noter tout d'abord que l'élaboration de ce programme a fait l'objet d'une large consultation de praticiens mais aussi de chercheurs, qui ont, pour certains d'entre eux, montré le risque d'une centration trop importante sur la langue, susceptible de contribuer à l'aggravation des inégalités scolaires (voir notamment Bautier, 2001). L'élaboration de ce programme a donc connu un déplacement de focale, depuis les dimensions normatives de la langue vers une meilleure prise en compte de ses usages proprement scolaires et des discours spécifiques des disciplines.

Mais ce changement de focale s'accompagne également d'un nouveau paradigme, pour reprendre la formule de Dominique Bucheton. On observe ainsi une présence forte de la notion de réflexivité, visant le développement de l'esprit critique : on relève notamment une quinzaine d'occurrences du terme réflexif et ses dérivés, dans des locutions variées, comme posture réflexive, démarche réflexive, pratiques réflexives, écrits réflexifs, et plus de 40 occurrences du terme réflexion. On note également la présence de plus de 60 occurrences du terme critique, dont la moitié dans la locution esprit critique: jugement critique, réflexion critique, sens critique, compréhension critique, discours critique, distance critique, recul critique etc.

Dans le B.O. de 2008, on note 3 occurrences de réflexion, sans lien avec les usages de la langue, aucune occurrence de réflexif ou de ses dérivés, ainsi que 3 occurrences de la locution esprit critique, en sciences, histoire-géographie et information-communication, mais pas en lien avec les usages de la langue, ici non plus. Même si le programme de 2015 est nettement plus volumineux que celui de 2008 , le nombre d'occurrences renvoyant au paradigme de la réflexivité et de l'esprit critique est donc proportionnellement bien plus présent dans le programme de 2015 .

\section{Des traces résiduelles d'une conception centrée sur la « maitrise de la langue »}

Pour autant, le "paradigme de la maitrise de la langue » nous semble encore très présent dans le programme de 2015. Si les locutions renvoyant à la correction formelle de la langue ont quasiment disparu, on observe cependant un nombre non négligeable d'occurrences concernant la notion de maitrise, dont 11 renvoyant directement à la maitrise de la langue, et une trentaine d'autres en lien indirect avec celle-ci, comme : maitriser les relations entre l'oral et l'écrit (p.17), maitrise du langage oral (p.18), maitriser l'écrit (p.18), maitriser la forme des mots en lien avec la syntaxe (p.104), maitriser une lecture orale et silencieuse fluide (p.109), maitriser les accords (p.119).

Étonnamment, on note que cette locution de maitrise de la langue n'apparait jamais dans ce programme quand il s'agit de langues étrangères ou régionales, où le terme de langue s'inscrit dans des formules comme entrée dans la langue, pratique de la langue, aspects culturels d'une langue, apprendre une langue, exposition à la langue, diversité des langues. La seule locution commune au français et aux langues étrangères et régionales désigne le fonctionnement de la langue. Le français est donc traité différemment, comme une langue à part - et il est vrai qu'elle a une fonction spécifique, conceptuelle - mais on peut supposer que les relations entre oral et écrit, par exemple, pourraient être un objet commun au français et aux autres langues. Par ailleurs, le terme de maitrise, réservé au seul français pour ce qui est des langues, apparait également pour d'autres disciplines, qui suggèrent que la notion de maitrise requiert une certaine technicité : on relève en mathématiques les formules maitrise des nombres et des opérations, maitrise de l'addition, de la soustraction (plus de 10 occurrences), en EPS ${ }^{4}$ les formules maitriser un affrontement collectif et individuel, maitriser les actions motrices, en Arts maitriser les codes, et dans le domaine du numérique, maitrise des techniques.

\footnotetext{
${ }^{4}$ EPS : éducation physique et sportive.
} 
Cette cohabitation discrète des paradigmes de la maitrise de la langue et de la réflexivité par le langage dans le programme de 2015 semble cependant augmentée par un autre, qui fait figure de paradigme émergeant : celui de l'explicitation, dont on peut penser d'un côté qu'elle peut être partie prenante de la réflexivité, puisque celle-ci suppose un certain recul, une distanciation par et avec le langage, pris explicitement pour objet ; mais la notion d'explicitation, en fonction des conceptions qui la sous-tendent, qu'il conviendra de mettre au jour, peut aussi renvoyer à la notion de transparence du langage. Or, cette idée d'un langage transparent s'inscrit pour nous dans la tradition de l'aristotélisme, qui privilégie l'exactitude, la classification en traits nécessaires et suffisants (Kleiber, 1990). Si cette classification en traits nécessaires et suffisants permet certes des distinctions précises et elles aussi nécessaires (entre évier et lavabo par exemple, ou, si l'on s'inscrit dans les disciplines scolaires, entre un trait et une droite), elle présente aussi le risque, pour les élèves, de croire en une illusoire coïncidence entre les mots et les choses (Authier-Revuz, 1984), qui viendrait écraser la possibilité de comprendre que le mot juste ne l'est pas dans l'absolu, mais relève d'un jugement, et plus précisément d'un jugement réflexif, en lien avec les apprentissages disciplinaires (Delarue-Breton, 2019). Le trait et la droite sont en effet des objets radicalement distincts, pour des raisons épistémiques et non pour des raisons de conformité à une norme sociale. Or, pour des raisons didactiques cette fois, les droites que rencontrent les élèves en mathématiques sont bien souvent représentées par des tracés constitués de traits, ce qui peut entrainer des confusions profondes, si ces tracés sont confondus durablement avec des droites, et non compris comme des représentations de cellesci.

\section{Les occurrences d'explicite dans le B.O. de 2015}

Avant de procéder à l'analyse des 61 occurrences du terme explicite ou de ses dérivés, il est cependant nécessaire de rappeler que l'explicitation, dont on verra qu'elle comprend des acceptions variées dans ce programme, suscite un certain engouement scientifique, sans doute pas étranger à l'intérêt que peut lui porter l'institution scolaire.

\section{Quelques remarques théoriques}

La notion d'explicitation en pédagogie fait l'objet, au plan théorique, de définitions distinctes, qui engagent des conceptions différentes non seulement de ce qui peut faire difficulté pour les élèves, mais aussi de ce qu'est l'apprentissage, et de ce que peut être le rôle du langage dans l'apprentissage. Pour aborder brièvement ce qui les distingue, rappelons tout d'abord que les deux modèles d'explicitation pédagogique que nous voulons évoquer attribuent à l'enseignant un rôle différent.

Pour les tenants de la Direct instruction, la pédagogie explicite, qui seule aurait fait ses preuves en matière d'enseignement efficace (Gauthier, Bissonette \& Richard, 2007), repose sur le principe que « l'enseignant est un facteur majeur d'explication de la variance scolaire » (op. cit. : 107), et que la pédagogie explicite est considérée comme efficace quelle que soit la discipline, et quel que soit le type d'élève, que celui-ci réussisse à l'école, ou soit plus en difficulté.

À ces considérations viennent se superposer, voire s'opposer celles des tenants d'une autre pédagogie explicite, dans la suite desquels nous nous inscrivons, susceptible de donner aux élèves eux-mêmes les moyens de la perception de ce qui est implicite (qui recouvre des objets de portées ou de niveaux fort différents) et les moyens de la compréhension des enjeux spécifiques des activités scolaires ; le rôle de l'enseignant est alors un rôle d'accompagnement d'une démarche d'apprentissage proprio motu (Brousseau, 1998) et non celui d'un modèle qui déplie tout ce qui peut l'être devant les élèves. Pour Rayou, «si le professeur peut bien 
expliciter les finalités d'un exercice, il ne peut expliciter tout ce qu'il en attend au risque de stériliser son intérêt et son pouvoir de développement des élèves » (Rayou, 2018 : 98).

Cet intérêt de l'exercice et ce pouvoir de développement peuvent être compris comme la marge de manœuvre du sujet apprenant, marge de manœuvre qui contribue aussi à la distinction entre tâche simple (application de procédures maitrisées) et tâche complexe, dont on considère qu'elle ne se réduit pas à une somme de tâches simples.

Pour Carette (2009) en effet, et nous le suivons largement sur ce point, une tâche complexe implique des élèves, pour être résolue, d'une part de maitriser des procédures (celles qui sont également nécessaires pour les tâches dites simples), d'autre part d'être capable de « choisir dans la situation proposée les traits pertinents à sa résolution » (op. cit. : 157). Du côté de l'enseignant, rendre cela possible suppose de « respecter un principe de réticence, au détriment d'une explicitation totale qui s'alimente vraisemblablement à une croyance néfaste dans la transparence des savoirs scolaires [...] » (Rayou, 2018 : 98). Rappelons enfin que certaines consignes très simples, qui permettent sans ambigüité de réaliser une tâche, ne révèlent pas les logiques sociales dans lesquelles elles s'inscrivent ou les enjeux qu'elles recèlent, phénomène qui relèverait plutôt, pour reprendre les propos de Bernstein (2007), du tacite que de l'implicite.

On comprend ainsi que s'il est sans doute nécessaire, d'une manière générale, de rendre par le langage plus transparentes qu'elles ne le sont aujourd'hui les conditions, visées et modalités des activités scolaires, il est nécessaire également de considérer que viser le « tout transparent », même sans risque de l'atteindre, oblitère une part conséquente des apprentissages scolaires, ceux qui nécessitent l'inventivité de la démarche.

L'intérêt, pour l'institution scolaire, de retenir le principe d'une pédagogie explicite qui viserait l'amélioration des pratiques pédagogiques et la volonté d'intégrer ce principe dans les programmes est donc sans aucun doute une position intéressante, mais à condition de donner aux enseignants les moyens de prendre la mesure de sa complexité théorique. Cette contribution a donc également pour objectif de rendre un peu plus perceptible l'épaisseur de la notion et l'hétérogénéité des orientations théoriques qu'elle sous-tend, et surtout d'en proposer, à partir de l'étude des emplois du terme et de ses dérivés dans ce programme, une vision plus structurée, qui lève un peu le voile sur la diversité de ses enjeux.

Notons cependant, car cette dimension est importante, que les deux approches que nous avons rapidement évoquées ont, bien qu'elles soient antagonistes, pour trait commun de considérer qu'on ne saurait imputer les apprentissages ou la réussite des élèves à leurs caractéristiques propres ou à celles de leur milieu, ce qui nous semble être une avancée dont on aimerait que l'école s'empare plus fermement.

\section{L'explicite dans le programme de 2015 : acceptions, occurrences et contextes d'emploi}

Précisons tout d'abord que parmi les 61 occurrences que nous avons étudiées dans le programme de 2015, 56 proviennent du programme pour l'école élémentaire (amputé du cycle 4 donc), et 5 du programme spécifique concernant l'école maternelle. Par ailleurs, le terme explicite(r) n'est pas absent du programme de 2008, qui comprend 9 occurrences (pour 39 pages), ce que nous aurions pu considérer comme proportionnellement comparable au nombre d'occurrences présentes dans le programme de 2015. Toutefois, le contexte d'emploi, qui représente le cœur de notre analyse, y est fort différent: dans le programme de 2008, 8 occurrences sur les 9 concernent le cycle 3 (la $9^{\text {ème }}$, située dans la présentation du programme, invite d'une manière globale à « proposer un enseignement explicite structuré, orienté vers les savoirs de base »), tandis que dans le programme de 2015, les occurrences concernent tous les cycles, y compris la maternelle, ce qui nous a amenée à parler de paradigme émergeant. Enfin, rappelons que ces chiffres sont donnés à titre indicatif, pour donner à voir des ordres de grandeur, mais des ordres de grandeur seulement : notre analyse ne se veut en aucun cas statistique. 
Le relevé systématique des termes formés autour de la base explicit-, à savoir les termes explicite (singulier ou pluriel), expliciter (infinitif, participe ou verbe conjugué), explicitement ou explicitation, a donné lieu à un classement, dont nous voudrions préciser qu'il ne se veut ni exhaustif ni exclusif, c'est-à-dire que d'autres catégories que celles que nous proposons peuvent être identifiées, et qu'un même terme peut relever de plusieurs catégories dans certains contextes d'emploi.

L'étude du programme de 2015 fait apparaitre que ce principe de l'explicitation, quelles que soient les acceptions du terme, est un principe général : il concerne tous les domaines disciplinaires, et tous les cycles, nous l'avons dit, y compris la maternelle. Nous avons donc regroupé les termes à partir de trois catégories principales, susceptibles de saisir l'ensemble des contextes d'emploi identifiés. La première catégorie renvoie à l'explicitation des procédures, stratégies et démarches sollicitées ou attendues à l'école, et dont nous montrerons qu'elles concernent aussi bien le point de vue des élèves que celui des enseignants. La deuxième catégorie renvoie à la transparence du contrat didactique et pédagogique. La troisième, quant à elle, regroupe les occurrences qui renvoient à la nécessité de comprendre les informations explicites d'un texte, mais aussi de rendre explicite l'implicite présent dans les textes, qu'il s'agisse de textes littéraires ou documentaires.

\section{Procédures, stratégies, démarches}

Du côté des enseignants, il s'agit d'abord d'enseigner explicitement des procédures valides, des manières de faire conformes aux principes disciplinaires. Ainsi, en mathématiques, les enseignants du cycle 2 sont invités à

Utiliser l'oral et l'écrit, le langage naturel puis quelques représentations et quelques symboles pour expliciter ${ }^{5}$ des démarches, argumenter des raisonnements. BO-74-C2 (mathématiques) ${ }^{6}$.

\section{En lecture,}

Afin de conduire chaque élève à une identification des mots sûre et rapide, des activités systématiques permettent d'installer et de perfectionner la maitrise du code alphabétique et la mémorisation des mots. Les démarches et stratégies permettant la compréhension des textes sont enseignées explicitement. BO-16-C2 (lecture).

Et pour ceux du cycle 3, il est indiqué que

Le langage oral trouve à se développer dans les dialogues didactiques, dans l'explicitation des démarches, dans les débats de savoirs ou d'interprétation [...]. BO-125-C3 (langage oral).

De plus,

Les démarches et stratégies permettant la compréhension des textes sont enseignées explicitement. Le cycle 3 développe plus particulièrement cet enseignement explicite de la compréhension afin de doter les élèves de stratégies efficaces et de les rendre capables de recourir à la lecture de manière autonome pour leur usage personnel et leurs besoins scolaires. BO-109-C3

\footnotetext{
${ }^{5}$ Nous soulignons systématiquement les occurrences d'explicite ou de ses dérivés dans les extraits du programme. ${ }^{6}$ Le référencement des extraits du programme est construit ainsi : BO pour Bulletin Officiel, 74 pour la page, C2 pour le cycle. La scolarité obligatoire française est organisée en 4 cycles : le premier concerne l'école maternelle (C1) ; le cycle 2, dit « cycle des apprentissages fondamentaux », recouvre les classes du CP (élèves de 6-7 ans, première classe de l'école élémentaire française) au CE2 (élèves de 8-9 ans); le cycle 3, dit "cycle de consolidation », recouvre les deux dernières classes de l'enseignement primaire (CM1 et CM2, élèves de 9-10 ans

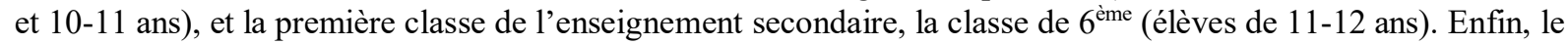
cycle 4, que nous n'avons pas étudié, dit «cycle des approfondissements », recouvre les 3 dernières années de collège, soit les classes de $5^{\text {ème }}, 4^{\text {ème }}$ et $3^{\text {ème }}$ (enfants de 12-13 ans, 13-14 ans et 14-15 ans).
} 
En mathématiques,

En CM1, le recours aux propriétés de linéarité (additive et multiplicative) est privilégié dans des problèmes mettant en jeu des nombres entiers. Ces propriétés doivent être explicitées; elles peuvent être institutionnalisées de façon non formelle à l'aide d'exemples [...]. BO-218-C3 (mathématiques)

Autrement dit, ces procédures, démarches et stratégies présentent un lien étroit avec le langage, médium privilégié de l'explicitation des savoir-faire disciplinaires ou relatifs aux domaines d'enseignement, quand il s'agit de l'école maternelle. Celui-ci n'a pour autant pas cette unique visée : il donne également aux élèves les moyens de l'autocontrôle de leur propre activité psychique, cognitive, intellectuelle.

Ainsi, en histoire-géographie,

Les élèves poursuivent au cycle 3 la construction progressive et de plus en plus explicite de leur rapport au temps et à l'espace, à partir des contributions de deux enseignements disciplinaires liés, l'histoire et la géographie. BO-170-C3 (HG).

En mathématiques, il s'agit d'être en mesure de

traiter des calculs relevant des quatre opérations, expliciter les procédures utilisées et comparer leur efficacité. BO-83-C2 (mathématiques).

Et pour l'écriture,

[...], les élèves prennent appui sur des réseaux de mots déjà constitués, convoquent ou recherchent les mots correspondant à l'univers de référence auquel fait appel la tâche d'écriture. Ils sont amenés à justifier explicitement le choix des mots utilisés et à les paraphraser. BO-118C3 (écriture)

Enfin, en ce qui concerne les élèves de l'école maternelle, il est précisé que

L'enseignant les incite à être précis pour comparer, différencier leurs points de vue et ceux des autres, émettre des questionnements : il les invite à expliciter leurs choix, à formuler ce à quoi ils pensent et à justifier ce qui présente à leurs yeux un intérêt. BO-11-C1 (langage oral).

D’une manière générale,

Dans tous les enseignements, et en particulier dans le champ "Questionner le monde», la familiarisation aux techniques de l'information et de la communication contribue à développer les capacités à rechercher l'information, à la partager, à développer les premières explicitations et argumentations et à porter un jugement critique. BO-12-C2 (outils transversaux).

L'explicitation langagière concerne donc simultanément les procédures pertinentes au sein des disciplines et les processus psychiques et cognitifs des élèves. Ces procédures doivent, selon ce programme, faire l'objet d'explicitation par les enseignants, afin de permettre aux élèves de les identifier, et de se les approprier.

L'explicitation vise cependant aussi à éclairer les attendus de la situation d'apprentissage, et est donc susceptible de contribuer à une certaine clarification des contrats, didactique (qui concerne la visée des activités en termes d'apprentissage) comme pédagogique (qui concerne davantage les modes de régulation de l'activité).

\section{Clarification du contrat didactique et pédagogique}

Ce programme fait tout d'abord apparaitre que la clarification du contrat didactique implique de passer d'activités qui sont, dans certaines disciplines ou certains domaines, intégrées à 
d'autres, à des activités dédiées, explicitement consacrées à l'apprentissage visé. Ainsi, en étude de la langue,

[...] le cycle 3 marque une entrée dans une étude de la langue explicite, réflexive, qui est mise au service des activités de compréhension de textes et d'écriture. BO-114-C3 (étude de la langue).

De même, pour l'oral,

Ces activités prennent place dans des séances d'apprentissage qui n'ont pas nécessairement pour finalité première l'apprentissage du langage oral mais permettent aux élèves d'exercer les compétences acquises ou en cours d'acquisition, et dans des séances de construction et d'entrainement spécifiques mobilisant explicitement des compétences de compréhension et d'expression orales. BO-105-C3 (langage oral).

Ou pour l'apprentissage du lexique :

Le lexique est pris explicitement comme objet d'observation et d'analyse dans des moments spécifiquement dédiés à son étude, et il fait aussi l'objet d'un travail en contexte, à l'occasion des différentes activités langagières et dans les différents enseignements. BO-119-C2 (étude de la langue).

Mais les enseignants sont conviés à aller au-delà de moments qui seraient seulement dédiés à tel ou tel apprentissage, et à clarifier les objets et enjeux de l'activité proposée. Ainsi, contrairement à la situation de M. Jourdain, qui fait de la prose sans le savoir, il est indiqué que

Les démarches et stratégies permettant la compréhension des textes sont enseignées explicitement. Deux éléments sont particulièrement importants pour permettre aux élèves de progresser : la répétition, la régularité voire la ritualisation d'activités langagières d'une part, la clarification des objets d'apprentissage et des enjeux cognitifs des tâches afin qu'ils se représentent ce qui est attendu d'eux d'autre part. BO-16-C2 (lecture).

À la fin du cycle 2, on considère que les élèves doivent être en mesure de

Pratiquer avec efficacité les formes de discours attendues - notamment, raconter, décrire, expliquer - dans des situations où les attentes sont explicites ; en particulier raconter seul un récit étudié en classe. $\mathrm{BO}-18-\mathrm{C} 2$ (langage oral).

Cette clarification des objets d'apprentissage est également prescrite à l'école maternelle :

L'enseignant rend lisibles les exigences de la situation scolaire par des mises en situation et des explications qui permettent aux enfants - et à leurs parents - de les identifier et de se les approprier. [...] Il aide à identifier les objets sur lesquels portent les apprentissages [...] faire expliciter par les enfants l'activité qui va être la leur. BO-3-C1.

La clarification des situations, nécessaire, n'est cependant pas considérée comme suffisante pour faire progresser les élèves : il s'agit également de clarifier les outils et des méthodes, mais aussi les règles collectives permettant l'organisation du travail; notamment, il y a lieu de favoriser

[l'] explicitation des repères pris pour comprendre (intonation, mots clés, connecteurs, etc.). BO14-C2 (langage oral).

Ou encore d'amener les élèves à la prise en compte de règles explicites établies collectivement. $\mathrm{BO}-15-\mathrm{C} 2$ (oral).

Enfin, il s'agit aussi de clarifier les critères d'évaluation : 
Prise en compte de critères d'évaluation explicites élaborés collectivement pour les présentations orales. BO-103-C3 (oral).

Y compris à l'école maternelle :

Les enseignants rendent explicites pour les parents les démarches, les attendus et les modalités d'évaluation propres à l'école maternelle. BO Mater-2-C1.

Ces différents cas de figure nous amènent à penser qu'il y a lieu de s'interroger sur la portée de cette exigence de clarification, qui peut s'entendre de deux manières radicalement opposées.

Soit il s'agit de viser la transparence du contrat, le «tout explicite », aussi bien en termes d'intention, d'objectifs visés que de moyens à mettre en œuvre pour y parvenir, dans une optique proche de celle de Gauthier et al. (2007), mais considérée comme illusoire et inappropriée par Brousseau (1998) et Rayou (2018). Soit il s'agit d'éviter les malentendus sociocognitifs, susceptibles de focaliser l'attention des élèves les moins familiers des modes de socialisation scolaire sur l'exécution de la tâche, au détriment de l'appropriation de ses enjeux didactiques (Bautier \& Rochex, 1997). Notre étude du programme de 2015 ne permet pas de faire pencher la balance d'un côté ou de l'autre, et il y a lieu de considérer que si la question de l'explicitation a paru un enjeu important pour ses concepteurs, la réflexion sur les deux modèles, ou du moins sur les deux approches qui la sous-tendent, n'y est pas spécifiquement prise en charge.

Par ailleurs, au-delà d'une plus grande transparence des contrats pédagogique et didactique, la notion d'explicitation concerne aussi la transparence des énoncés.

\section{Explicite et implicite dans les textes et documents}

Plus exactement, la question de l'explicitation est fortement impliquée dans les activités qui visent la compréhension des textes et documents, dans tous les cycles, et dans l'ensemble des domaines disciplinaires: la préoccupation de l'institution est récurrente sur ce point. Notamment, il s'agit en Langue vivante au cycle 2 de permettre aux élèves, à l'issue du cycle, de comprendre déjà ce qui est explicite, à partir d'une

Appréhension individuelle du document sonore et mises en commun pour repérer et restituer son sens explicite, sans s'interdire le recours à la langue française si besoin. BO-37-C2 (langue vivante).

Mais il s'agit également de comprendre ce qui n'est pas explicite :

L'apprentissage de la lecture nécessite aussi de comprendre des textes narratifs ou documentaires, de commencer à interpréter et à apprécier des textes, en comprenant ce qui parfois n'est pas tout à fait explicite. BO-4-C2 (lecture).

Nous notons en passant la formule un peu surprenante « pas tout à fait », qui laisse entendre qu'au cycle 2 , les élèves ne seraient jamais confrontés à des textes comprenant des passages tout à fait implicites, dont le sens est à construire...

Enfin, pour les plus jeunes, il s'agit d'être en mesure de parler de ce qui n'est pas présent, autrement dit d'évoquer des objets absents :

L'école demande régulièrement aux élèves [...] de parler de ce qui n'est pas présent [...]. Ces situations offrent [...] un moyen de s'entrainer à s'exprimer de manière de plus en plus explicite. BO-Mater-6-C1. 


\section{Expliciter quoi ? Quelques éléments de discussion}

Nous avons fait à dessein le choix d'analyser des extraits du programme de 2015 comprenant au moins une occurrence de l'un des termes formés à partir de la base explicit-, afin de travailler sur un corpus restreint ; cette étude met au jour une hétérogénéité d'usages, et une hétérogénéité des positionnements attendus en matière d'explicitation, de la part des élèves comme des enseignants, dont la juxtaposition est susceptible de brouiller les visées spécifiques.

Dans certains cas, il s'agit en effet de rendre explicites, dans le discours pédagogique, les procédures, démarches ou raisonnements pertinents dans les différentes disciplines scolaires ; dans d'autres, il s'agit d'amener les élèves eux-mêmes à une posture métalangagière destinée à favoriser l'autorégulation des apprentissages ; dans d'autres cas encore, de clarifier les contrats didactique et pédagogique ; enfin, il s'agit également d'apprendre aux élèves à rendre explicite ce qui ne l'est pas dans les documents qui leur sont proposés.

Or, ces différentes attentes ne convoquent pas les mêmes postures, en particulier langagières, et engagent différentes conceptions de la langue et de ses usages, en matière de réflexivité notamment.

Authier-Revuz rappelle en effet que la réflexivité du langage humain, c'est-à-dire sa capacité à se prendre lui-même pour objet, qui s'inscrit dans la langue elle-même à travers l'autonymie, quand un même mot désigne simultanément un référent objectal et le mot lui-même, peut être perçue de deux manières différentes. D'un côté, dans la tradition aristotélicienne, elle fait figure de " défaut des langues naturelles », susceptible de " perturber le calcul du vrai », quand un mot peut désigner deux objets distincts. De l'autre, par exemple pour Benveniste ou Wittgenstein, la réflexivité apparait au contraire comme une propriété spécifique des langues naturelles, leur conférant un pouvoir majeur que n'offrent pas d'autres systèmes de signes (Authier-Revuz, 2003 : 67). De ce point de vue, on peut considérer que c'est la réflexivité naturelle de la langue qui rend possible la réflexivité par le langage.

On retrouve, selon nous, cette ambivalence entre une langue entendue comme devant être exacte vs une langue entendue comme espace de négociation dans l'emploi de la notion d'explicitation dans le programme de 2015.

D'un côté, expliciter vise en effet à désambigüiser ${ }^{7}$ le propos, donc à lui conférer une certaine univocité, et à faire coïncider, en quelque sorte, les mots et les choses; par exemple quand il s'agit de clarifier les contrats, il est attendu des élèves qu'ils perçoivent de manière univoque les attendus de l'activité proposée, en termes de réalisation de la tâche comme en termes d'enjeux didactiques notamment. Autre exemple, quand il s'agit de s'accorder sur le sens d'un terme ou d'un passage d'un texte ou document, qui ne donne pas lieu à interprétation : la table de multiplication désigne un tableau, et non un meuble.

De l'autre, il s'agit au contraire de ménager pour les élèves une marge de manœuvre, c'està-dire un espace de déambulation (Delarue-Breton \& Crinon, 2016), visant à favoriser la prise de distance et une forme de réflexivité vis-à-vis de l'objet étudié. Par exemple, lorsqu'il s'agit de produire des énoncés concourant à expliciter des textes ou documents dont le sens est à construire, et non donné, il est attendu des élèves qu'ils perçoivent qu'il n'y a pas, pour reprendre la formulation d'Authier-Revuz, de coïncidence entre les mots et les choses, ni même des mots à eux-mêmes ou encore du discours à lui-même (Authier-Revuz, 2003 : 91-93), et que c'est précisément cet écart entre les mots et les choses, entre les mots eux-mêmes ou entre le discours et lui-même qui ménage un espace de réflexivité par le langage. Par exemple, quand il s'agit de distinguer le théâtre comme lieu du théâtre comme genre littéraire.

Notons cependant que cette double dimension du processus d'explicitation reflète pour nous non pas deux conceptions opposées, mais bien deux aspects de ce processus, l'espace de

\footnotetext{
${ }^{7}$ Nous empruntons également ce terme à Authier-Revuz (2003).
} 
déambulation étant susceptible de permettre à chacun d'accéder à une construction de significations pertinentes, pour construire une intersubjectivité partagée, permettant elle-même l'élaboration d'un corps commun - et non singulier - de catégories de pensée.

Pour rendre compte de cette hétérogénéité des usages - et par conséquent, des acceptions des termes ayant pour base explicit- dans le programme de 2015, nous formulons l'hypothèse qu'elle est peut-être, du moins pour partie, le reflet de la complexité des positions scientifiques au sujet de l'explicitation en contexte scolaire, qui ne sont pas neutres. Il nous parait dès lors intéressant de tenter d'établir un rapprochement entre conceptions institutionnelles et conceptions scientifiques.

Si l'on reprend rapidement les principales propositions de la pédagogie explicite au sens de la Direct instruction, il s'agit pour les enseignants de dire, montrer et guider (Gauthier et al., 2007).

Dire signifie pour la Direct Instruction « rendre explicites pour les élèves les intentions et objectifs visés dans la leçon », mais aussi « rendre explicites et disponibles pour les élèves les connaissances antérieures dont ils auront besoin ». De ce point de vue, les contenus évoqués dans notre catégorie 2 (Clarification des contrats didactique et pédagogique) sont susceptibles de participer du dire ainsi défini, du moins dans sa première partie (la question des connaissances antérieure est peu évoquée dans le programme, et concerne exclusivement le français, aux cycles 2 et 3 ).

Rappelons cependant d'une part que clarifier le contrat didactique ne consiste pas nécessairement, nous le redisons, à le rendre totalement transparent, au risque de placer l'élève en situation d'exécution de tâche ; précisons d'autre part que ces contenus laissent dans l'ombre la manière dont on peut interpréter les situations scolaires (Bautier \& Rochex, 1997), et notamment les enjeux spécifiques de la socialisation scolaire.

Montrer signifie ici «rendre la demande explicite pour les élèves en exécutant devant eux la tâche à accomplir et en énonçant le raisonnement suivi à voix haute ». Cette dimension n'est pas présente dans le programme, et nous notons par ailleurs qu'elle exclut la possibilité pour l'élève, si cette manière de montrer est systématique, d'élaborer un cheminement propre, autrement dit de choisir les procédures à mobiliser dans telle ou telle situation, qui sont des compétences visées par le programme (les élèves du cycle 2 doivent par exemple être en mesure d' « élaborer ou choisir des stratégies de calcul »; BO-83-C2). On peut donc considérer qu'il y a une cohérence entre cette absence et les préconisations du programme, mais on note également que cette cohérence est implicite.

Enfin, guider signifie «chercher à ce que les élèves rendent explicite leur raisonnement implicite en situation de pratique [...]»: les contenus abordés dans notre catégorie 1 (Procédures, stratégies, démarches), dont nous rappelons qu'elle concerne les enseignants comme les élèves, participent sans doute pour partie de ce guidage, qui invite les enseignants à expliciter les procédures attendues, et à les faire expliciter par les élèves.

Cependant, ces postures attendues de l'enseignant dans la théorie de la Direct instruction, et auquel le programme de 2015 fait écho pour partie, ne concernent que la maitrise des procédures, qui n'est qu'une des composantes nécessaires pour être en mesure de résoudre des tâches complexes. Carette précise en effet ceci :

Les résultats de notre recherche nous ont permis de conclure que si la plupart des élèves maitrisaient des procédures, un nombre important était incapable de les mobiliser. En d'autres termes, ils présentaient des difficultés à "cadrer 》la situation. (Carette, 2009 : 159)

Autrement dit, ce qui distingue les élèves qui parviennent à mobiliser les ressources nécessaires à la résolution d'une tâche complexe de ceux qui n'y parviennent pas, et donc ce qui est au cœur des inégalités scolaires, c'est pour l'auteur la capacité à identifier les traits 
pertinents à la résolution d'une tâche complexe (ce qu'il nomme le cadrage), qui permet en effet aux élèves de mobiliser les ressources dont il dispose ; or, cette capacité s'apprend, c'est précisément là, pensons-nous, le rôle de la socialisation scolaire.

Carette (2009 : 157) distingue trois types de cadrage : le cadrage hyperpragmatique, quand les élèves résolvent des tâches sans tenir compte de l'enjeu scolaire qui les sous-tend, le cadrage hyperscolaire, qui consiste à utiliser une procédure automatisée apprise sans se poser la question de sa pertinence en contexte, et le cadrage scolaire ou instruit, qui suppose à la fois la maitrise des procédures et la capacité à identifier les traits pertinents à la résolution de la tâche.

On pourrait considérer que, finalement, la maitrise des procédures pourrait suffire, et que la visée de l'école primaire n'est pas de former les élites. Notre position est cependant très différente.

Nous considérons en effet que l'école est précisément le lieu où doivent se réduire les inégalités, et non le lieu où elles peuvent continuer à s'accroitre. C'est donc à l'école que doit s'apprendre et se développer, a fortiori si ce n'est pas fait par ailleurs, la capacité à identifier les traits pertinents à la résolution de toutes sortes de tâches, y compris complexes, sans quoi certains élèves courent le risque, pour reprendre la formule de Bernstein (2007 : 36) d'être «positionnés dans un monde factuel lié aux opérations simples, et où le savoir est imperméable ».

\section{Pour conclure}

Pour que l'école devienne ce lieu où les inégalités se réduisent, il nous apparait nécessaire pour l'institution scolaire d'en clarifier les enjeux linguistiques et langagiers, en assumant les tensions - légitimes - qui les sous-tendent.

L'étude du programme de 2015 montre en effet que les emplois de termes formés à partir de la base explicit- traduisent, mais sans la révéler, la tension entre une double nécessité paradoxale, transparence du langage d'un côté, et réflexivité par le langage de l'autre, qui s'inscrit directement dans la suite de la tension que nous avons évoquée au cours de cet article entre maitrise de la langue et langage réflexif. Au-delà du sens inscrit dans les énoncés, et donc dans la langue, s'élaborent des significations qui sont, pour reprendre la formule de Bronckart (1987), le produit de l'usage verbal, et donc du langage. Il s'agit donc d'un côté de faire disparaitre les zones d'ombre que recèlent potentiellement les énoncés, ou les situations, de les rendre univoques, sans ambigüité ; de l'autre, il s'agit d'exploiter - et pour les élèves, d'explorer - la pluralité des significations produites dans le langage et dans les situations, pour induire une réflexivité susceptible de contribuer à la secondarisation de l'expérience première, autrement dit de leur permettre de revisiter cette expérience à l'aune des catégories de pensée des disciplines scolaires.

La récurrence d'emploi du terme explicite et de ses dérivés dans ce programme ne saurait donc être un mot d'ordre en soi, une règle à suivre en toute occasion et en toute circonstance ; la notion même d'explicitation implique l'idée d'un processus, qui peut être tantôt le moyen de l'activité scolaire, quand il s'agit de bien comprendre les conditions de réalisation de la tâche par exemple, ou ses visées, tantôt la finalité même de l'activité, quand il s'agit de construire le sens d'un texte ou document implicite par exemple.

Cependant, si nous considérons qu'il n'est pas opportun de déplier de manière systématique les tenants et les aboutissants de toute activité scolaire, ce qui pourrait non seulement contrevenir à l'élaboration d'un cheminement propre des élèves, mais encore et surtout oblitérer la possibilité d'apprendre à faire des choix pertinents, nous considérons également que la non- 
perception in fine des enjeux d'apprentissage de ces activités par tous les élèves est une source puissante d'inégalité, et en particulier lorsqu'il s'agit de l'activité langagière.

Il nous apparait donc important que les enseignants puissent focaliser l'activité langagière des élèves au moins autant sur l'outil cognitif puissant que représente le langage, pour reprendre la formule de Ramognino (2007), que sur les normes structurelles de la langue.

Plus largement, il y a lieu de donner la possibilité aux élèves de comprendre que le dialogue scolaire, constitué des échanges entre enseignant et élèves en classe, s'inscrit dans un double mouvement, constitué d'une part de temps d'élaboration des significations et de négociation du sens, dans un espace de réflexivité fait d'approximations successives, d'autre part de temps de stabilisation des significations, permettant de poser les propriétés des savoirs conceptuels disciplinaires (Delarue-Breton, 2019).

Du côté des normes, substituer à une conception normalisante de la langue, relevant de l'imposition sociale, une conception normative, centrée sur les échanges langagiers permettant les apprentissages disciplinaires, donnerait à l'enseignant l'occasion d'être «[...] un être normatif, à qui une société confie de se porter garant de la normativité intellectuelle, de la pensée vivante, vivant de connaissances et du désir de connaitre, des nouveaux venus » (Cornu, $2009: 42)$.

Enfin, nous souhaitons mentionner qu'il nous semble dommageable que les apports de la recherche en éducation fassent l'objet, du côté de l'institution scolaire, d'un mouvement de balancier qui, au gré des évolutions politiques, privilégie de manière souvent exclusive tantôt tel type d'apport, tantôt tel autre, au lieu de s'inscrire dans une perspective cumulative.

\section{Bibliographie}

AUTHIER-REVUZ Jacqueline, 1984, « Hétérogénéité(s) énonciative(s) », Langages, n73, p. 98-111.

AUTHIER-REVUZ Jacqueline, 2003, «Le Fait autonymique : langage, langue, discours. Quelques repères », dans J. Authier-Revuz, M. Doury \& M. Reboul-Touré (dir.), Parler des mots - Le fait autonymique en discours, Paris, Presses de la Sorbonne nouvelle, p. 67-96.

BAUTIER Élisabeth, 2001, "Pratiques langagières et scolarisation », Revue française de pédagogie, $\mathrm{n}^{\circ} 137$, p. 117-161.

BAUTIER Élisabeth, RAYOU Patrick, 2009, Les inégalités d'apprentissage. Programmes, pratiques et malentendus scolaires, Paris, Presses Universitaires de France.

BAUTIER Élisabeth, ROCHEX Jean-Yves, 1997, "Ces malentendus qui font les différences », dans Jean-Pierre Terrail (Éd.), La scolarisation en France, critique de l'état des lieux, Paris, La Dispute, p. 105-122.

BERNSTEIN Basil, 2007, Pédagogie, contrôle symbolique et identité - Théorie, recherche, critique, Laval, Les presses de l'université Laval.

BRONCKART Jean-Paul, 1987, «Interaction, discours, significations », Langue française, $n^{\circ} 74$, p. 29-50.

BROUSSEAU Georges, 1998, Théorie des situations didactiques, Grenoble, La Pensée Sauvage.

BUCHETON Dominique (dir.), 2014, Refonder l'enseignement de l'écriture. Vers des gestes professionnels plus ajustés du primaire au lycée, Retz, Paris.

CARETTE Vincent, 2009, «Et si on évaluait des compétences en classe ? À la recherche du "cadrage instruit" ", dans Lucie Mottier Lopez et al., Évaluations en tension, Bruxelles, De Boeck Supérieur, p. 147-163. 
CORNU Laurence, 2009, « Normalité, normalisation, normativité : pour une pédagogie critique et inventive ", Le Télémaque, n³6, p. 29-44.

DELARUE-BRETON Catherine, 2019, « Le dialogue scolaire, un genre discursif frontalier », Raisons éducatives, $\mathrm{n}^{\circ}$ 23, p. 47-69. [En ligne] : https://www.cairn.info/revue-raisonseducatives-2019-1-page-47.htm

DELARUE-BRETON Catherine, 2012, Discours scolaire et paradoxe, Louvain, AcademiaL'Harmattan.

DELARUE-BRETON Catherine, CRINON Jacques, 2016, «Circulation, déambulation et textes hétérogènes », Recherches en Éducation, $\mathrm{n}^{\circ} 25$, p. 34-45.

GAUTHIER Clermont, BISSONNETTE Steve, RICHARD Mario, 2007, Chapitre 7, l'enseignement explicite, dans Vincent Dupriez et Gaëtane CHAPELLE, Enseigner, Paris, PUF, p. 107-116. [En ligne, consulté le 6 mai 2020]: http://www.formapex.com/telechargementpublic/gauthier2007c.pdf

KLEIBER Georges, 1990, La sémantique du prototype, Paris, PUF.

MINISTÈRE DE L'ÉDUCATION NATIONALE, DE L'ENSEIGNEMENT SUPÉRIEUR ET DE LA RECHERCHE, 2008, «Horaires et programmes d'enseignement de l'école primaire », Bulletin Officiel Hors-série $n^{\circ} 3$ du 19 juin 2008.

MINISTÈRE DE L'ÉDUCATION NATIONALE ET DE LA JEUNESSE, 2018a, «Enseignements primaire et secondaire, Programmes d'enseignement, Annexe 1 (cycle des apprentissages fondamentaux : cycle 2) », Bulletin Officiel $n^{\circ} 30$ du 26 juillet 2018.

MINISTÈRE DE L'ÉDUCATION NATIONALE ET DE LA JEUNESSE, 2018b, «Enseignements primaire et secondaire, Programmes d'enseignement, Annexe 2 (cycle de consolidation : cycle 3) », Bulletin Officiel $n^{\circ} 30$ du 26 juillet 2018.

MINISTÈRE DE L'ÉDUCATION NATIONALE ET DE LA JEUNESSE, 2015, « Programmes d'enseignement du cycle des apprentissages fondamentaux (cycle 2), du cycle de consolidation (cycle 3 ) et du cycle des approfondissements (cycle 4) », Bulletin Officiel Spécial $n^{\circ} 11$ du 26 novembre 2015.

PELLAT Patrick, 2017, «Les programmes de 2015: des avancées grammaticales et didactiques », Le Français aujourd'hui, n 198 , p. 15-24.

RAMOGNINO Nicole, 2007, "Normes sociales, normativités individuelle et collective, normativité de l'action », Langage et société, n¹19, p.13-41.

RAYOU Patrick, 2018, «Pédagogie explicite », Recherche \& Formation, n॰87, p.97-107. [En ligne, consulté le 6 mai 2020] : https://www.cairn.info/revue-recherche-et-formation2018-1-page-97.htm

ROCHEX Jean-Yves, CRINON Jacques, 2011, La construction des inégalités scolaires. Au cour des pratiques et des dispositifs d'enseignement, Rennes, Presses Universitaires de Rennes. 


\section{GLOTTOPOL}

Revue de sociolinguistique en ligne

Comité de rédaction : Michaël Abecassis (University of Oxford), Salih Akin (Université de Rouen Normandie), Sophie Babault (Université de Lille), Aude Bretegnier (Université du Mans), Claude Caitucoli, Véronique Castellotti (Université de Tours), Régine DelamotteLegrand (Université de Rouen Normandie), Alexandre Duchêne (Université de Fribourg), Valentin Feussi (Université d'Angers), Robert Fournier (Carleton University, Ottawa), Stéphanie Galligani (Université Grenoble Alpes), Médéric Gasquet-Cyrus (Université AixMarseille), Emmanuelle Huver (Université de Tours), Normand Labrie (Université de Toronto), Foued Laroussi (Université de Rouen Normandie), Benoit Leblanc (Université du Québec à Trois-Rivières), Mylène Lebon-Eyquem (Université de la Réunion), Fabienne Leconte (Université de Rouen Normandie), Gudrun Ledegen (Université de Rennes), Danièle Moore (Simon Fraser University, Vancouver), Clara Mortamet (Université Jean Monnet, Saint Etienne), Alioune Ndao (Université Cheik Anta Diop, Dakar), Isabelle Pierozak (Université de Tours), Cécile Van den Avenne (Université Sorbonne Nouvelle, Paris 3).

Rédactrice en chef : Clara Mortamet.

Directrice de publication : Fabienne Leconte.

Comité scientifique: Michelle Auzanneau (Université de Paris), Margaret Bento (Université de Paris), Jacqueline Billiez (Université de Grenobles Alpes), Philippe Blanchet (Université de Rennes), Jean-Michel Eloy (Université d'Amiens), Françoise Gadet (Université Paris Nanterre), Monica Heller (Université de Moncton), Caroline Juillard (Université de Paris), Jean-Marie Klinkenberg (Université de Liège), Marinette Matthey (Université Grenoble Alpes), Marie-Louise Moreau (Université de Mons-Hainault), Robert Nicolaï (Université Côte d'Azur), Didier de Robillard (Université de Tours), Valérie Spaëth (Université Sorbonne nouvelle), Claude Truchot (Université de Strasbourg), Daniel Véronique (Université AixMarseille).

\section{Comité de lecture pour ce numéro :}

Nathalie Auger, Michèle Auzanneau, Margaret Bento, Stéphane Bonnery, Josiane Boutet, Lucile Cadet, Danièle Cogis, Claudine Garcia-Debanc, Marc Debono Régine Delamotte, JeanFrançois De Pietro, Marie-Laure Elalouf, Valentin Feussi, Laurent Gajo, Emmanuelle Huver, Christian Lagarde, Mylène Lebon-Eyquem, Fabienne Leconte, Gudrun Ledegen, Nadia Maillard, Maira Mamede, Bruno Maurer, Clara Mortamet, Fanny Rink, Valerie Spaëth, Marielle Rispail, Françoise Ropé, Eguzki Urteaga, Cécile Van den Avenne, Daniel Véronique 